\title{
Prenatal nicotine exposure enhances the susceptibility to metabolic syndrome in adult offspring rats fed high-fat diet via alteration of HPA axis-associated neuroendocrine metabolic programming
}

\author{
Dan XU $U^{1,2, \#, ~ L i-p i n g ~ X I A ~}{ }^{1, \#}$, Ben-jian ZHANG ${ }^{1}$, Lang SHEN ${ }^{1}$, You-ying LEI ${ }^{1}$, Lian LIU $^{1}$, Li ZHANG $^{1}$, Jacques MAGDALOU ${ }^{3}$, \\ Hui WANG ${ }^{1,2, *}$ \\ ${ }^{1}$ Department of Pharmacology, Basic Medical School of Wuhan University, Wuhan 430071, China; ${ }^{2}$ Research Center of Food and Drug \\ Evaluation, Wuhan University, Wuhan 430071, China; ${ }^{3}$ UMR 7561 CNRS-Nancy Université, Faculté de Médecine, Vandoeuvre-lès- \\ Nancy, France
}

Aim: Prenatal nicotine exposure (PNE) alters the hypothalamic-pituitary-adrenocortical (HPA) axis-associated neuroendocrine metabolic programming in intrauterine growth retardation offspring rats. In this study we aimed to clarify the susceptibility to metabolic diseases of PNE offspring rats fed a high-fat diet.

Methods: Maternal Wistar rats were injected with nicotine $(1.0 \mathrm{mg} / \mathrm{kg}, \mathrm{sc})$ twice per day from gestational day 11 until full-term delivery, and all pups were fed a high-fat diet after weaning and exposed to unpredictable chronic stress (UCS) during postnatal weeks 18-20. Blood samples were collected before and after chronic stress, and serum ACTH, corticosterone, glucose, insulin, total cholesterol, triglyceride and free fatty acids levels were measured. The hypothalamus, pituitary gland and liver were dissected for histological studies.

Results: UCS significantly increased the serum ACTH, corticosterone and insulin levels as well as the insulin resistant index without changing the serum glucose, total cholesterol, triglyceride and free fatty acids levels in adult offspring rats without PNE. The body weight of PNE offspring rats presented a typical "catch-up" growth pattern. PNE not only aggravated the UCS-induced changes in the HPA axis programmed alteration (caused further increases in the serum ACTH and corticosterone levels), but also significantly changed the glucose and lipid metabolism after UCS (caused further increases in the serum glucose level and insulin resistant index, and decrease in the serum free fatty acids). The effects of PNE on the above indexes after UCS showed gender differences. Pathological studies revealed that PNE led to plenty of lipid droplets in multiple organs.

Conclusion: PNE enhances not only the HPA axis, but also the susceptibility to metabolic diseases in adult offspring rats fed a high-fat diet after UCS in a gender-specific manner and enhances the susceptibility to metabolic diseases in adult offspring rats fed a high-fat diet.

Keywords: nicotine; intrauterine growth retardation; hypothalamic-pituitary-adrenal axis; neuroendocrine metabolic programming; highfat diet; stress; metabolic syndrome; insulin; glucose; lipid; gender difference

Acta Pharmacologica Sinica (2013) 34: 1526-1534; doi: 10.1038/aps.2013.171; published online 25 Nov 2013

\section{Introduction}

Metabolic syndrome (MS) is a collection of multiple conditions, including hypertension, hyperglycemia, dyslipidemia and obesity, that directly lead to fatty liver and diabetes, as

\footnotetext{
\# These authors contributed equally to this work.

* To whom correspondence should be addressed.

E-mail wanghui19@whu.edu.cn

Received 2013-08-16 Accepted 2013-10-22
}

well as cardiovascular and cerebrovascular diseases. Previous reports showed that in adults over the age of 20, the prevalence of metabolic syndrome is 34\%-39\% in the United States ${ }^{[1]}$, and the morbidity is as high as $14 \%-16 \%$ in China ${ }^{[2]}$. Intrauterine growth retardation (IUGR) refers to the poor growth of a baby in the womb during pregnancy. Specifically, it is defined as a developing baby who weighs $10 \%$, or two standard deviations, less than the mean body weight of other babies of the same gestational age ${ }^{[3]}$. The worldwide 
prevalence rate for IUGR is approximately $3 \%-16 \%{ }^{[4]}$. Epidemiological investigations have indicated that the incidence of adult MS in IUGR fetuses is 5.75-fold higher than in normal fetuses $^{[5]}$. All of these above-mentioned reports indicate that MS has a fetal origin.

Intrauterine programming is the process by which the structure and function of tissues are permanently altered by insults suffered during early development ${ }^{[6]}$. To date, the underlying mechanisms of susceptibility to MS in adult offspring with IUGR have not been clearly defined. They might arise from functional developmental alterations of the hypothalamicpituitary-adrenocortical (HPA) axis, which inhibits fetal growth and increases the sensitivity of peripheral tissues to metabolic hormones, such as glucocorticoids $(\mathrm{GC})^{[7]}$. Because the postnatal nutrient availability is better than that predicted prenatally, the offspring exhibit "catch-up" growth and fat deposition with an increased risk of adult insulin resistance $(\mathrm{IR})^{[7]}$. Furthermore, the enhanced sensitivity of the HPA axis in response to stress in adult offspring could accelerate the development of metabolic abnormalities.

Cigarette smoke contains various compounds that may be harmful to the developing fetus. Nicotine is one of these adverse components ${ }^{[8]}$. It is known to cross the placenta and can be detected in fetal circulation, where it can affect the fetus in several ways ${ }^{[9-12]}$. Our previous studies ${ }^{[13]}$ found that prenatal nicotine exposure (PNE) caused IUGR in rats; the fetuses were overexposed to maternal GC, and the development of the fetal HPA axis was inhibited. Meanwhile, the metabolic pathways for glucose and lipids in the fetal liver and gastrocnemius muscle, as well as the corresponding blood phenotypes, were affected, which could most likely be attributed to the increased fetal circulatory GC level. After birth and the initiation of a normal diet, the IUGR offspring induced by PNE showed low basal activity but an enhanced sensitivity of the HPA axis to chronic stress, as well as GC-dependent phenotypic alterations of the blood glucose and lipid metabolism ${ }^{[14]}$. These results suggest an underlying mechanism of "HPA axisassociated neuroendocrine metabolic programmed alteration" in adult IUGR offspring with PNE-induced susceptibility to MS.

A high-fat diet has been linked to hypertension, glucose intolerance, insulin resistance, type 2 diabetes, dyslipidemia, obesity and reproductive disorders in adults ${ }^{[15-18]}$. A key question is whether PNE (which induces the HPA axis-associated neuroendocrine metabolic programmed alteration) increases the susceptibility to adult MS and associated metabolic diseases in adult offspring rats fed a high-fat diet. However, it remains unknown whether gender plays a role in the effects observed in adult IUGR offspring. To address these questions, a high-fat diet and unpredictable chronic stresses ${ }^{[19]}$ were employed to simulate good nutrition and physical stress in the present study, and we observed the function of the HPA axis, the profiles of glucose and lipid metabolism, and the morphological changes of multiple organs, in addition to gender differences in the adult IUGR offspring induced by PNE. This study may help elucidate the underlying mechanisms respon- sible for the susceptibility of IUGR offspring to adult metabolic diseases.

\section{Materials and methods Materials}

Nicotine (CAS No $54-11-5$, with a purity of 98\%) was provided by Sanqiang Co, Ltd (Weifang, China). Isoflurane was purchased from Baxter Healthcare Co (Deerfield, IL, USA). Oligonucleotide primers were synthesized by Sangon Biotech Co, Ltd (Shanghai, China). Adrenocorticotropic hormone (ACTH) and insulin radioimmunoassay kits were purchased from the North Institute of Biological Technology (Beijing, China). A rat corticosterone (CORT) ELISA kit was obtained from Assaypro LLC (Saint Charles, MO, USA). A glucose oxidase assay kit was provided by Mind Bioengineering Co, Ltd (Shanghai, China). A total cholesterol (TCH) and triglyceride (TG) assay kit and a free fatty acid (FFA) assay kit were purchased from Sangon Biotech Co, Ltd (Shanghai, China). Other chemicals and agents were of analytical grade.

\section{Animals and treatment}

Specific pathogen-free (SPF) Wistar rats (weighing 200-240 g for females and 260-300 $\mathrm{g}$ for males) were obtained from the Experimental Center of Hubei Medical Scientific Academy (No 2008-0005, Wuhan, China). Animal experiments were performed at the Center for Animal Experiment of Wuhan University (Wuhan, China), which has been accredited by the Association for Assessment and Accreditation of Laboratory Animal Care International (AAALAC International). All experimental procedures involving animals were approved by and performed in accordance with the Guidelines for the Care and Use of Laboratory Animals of the Chinese Animal Welfare Committee.

Animals were held under temperature-controlled conditions on a 12-h light-dark cycle and had ad libitum access to standard chow and tap water. After one week of acclimation, two females were mated with one male for one night. Upon confirmation of mating by the observation of sperm in a vaginal smear, the day was noted as gestational day 0 (GD0). Pregnant females were then transferred to individual cages.

The rat IUGR model was induced by PNE as previously reported $^{[13,14]}$. A schematic of the procedure for maternal and offspring rat treatment was shown in Figure 1. Pregnant rats were randomly divided into two groups: a control group and a nicotine group. Starting from GD11 until term delivery (GD21), the nicotine group was subcutaneously administered with $1.0 \mathrm{mg} / \mathrm{kg}$ nicotine twice per day. The control group was given the same volume of saline. At parturition, the dams and their offspring were fed ad libitum.

On postnatal day 1 (PD1), 8 pups were selected randomly from each mother and their gender ratio was balanced to 1:1 to ensure adequate and standardized nutrition until weaning ${ }^{[20]}$. At postnatal week 4 (PW4), 16 pups from 8 different mothers were selected randomly for each group ( 8 male and 8 female IUGR pups from the nicotine group, 8 male and 8 female normal pups from the control group), and all pups were fed 


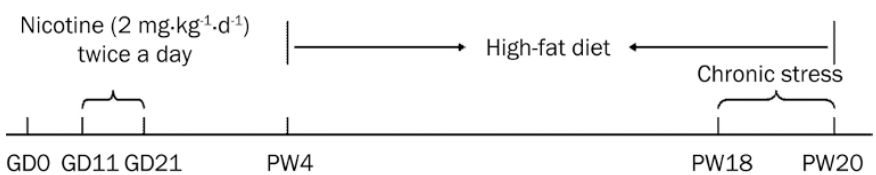

Figure 1. The schedule of animal treatment from gestational day 0 (GDO) to postnatal week 20 (PW20).

a high-fat diet ad libitum before being sacrificed. Standard rodent chow (purchased from the Experimental Center of Hubei Medical Scientific Academy) provided 21\% kcal from protein, $68.5 \% \mathrm{kcal}$ from carbohydrates, and only $10.5 \% \mathrm{kcal}$ from fat. The high-fat diet was previously reported by our $\mathrm{lab}^{[21]}$ to contain $88.0 \%$ corn flour, $11.5 \%$ lard, and $0.5 \%$ cholesterol, which provided $18.9 \% \mathrm{kcal}$ from protein, $61.7 \% \mathrm{kcal}$ from carbohydrate, and $19.4 \% \mathrm{kcal}$ from fat.

The offspring rats were weighed weekly. The rate of body weight growth was calculated as follows: Gain rate of body weight $(\%)=[($ body weight at PWX-body weight at PW1)]/ body weight at PW1 $\times 100$. At PW16, the animals were fasted for $12 \mathrm{~h}$, and blood was taken via the vena caudalis within 3 min to measure levels of ACTH, CORT, glucose, insulin, TCH, TG and FFA. Starting at PW18, the rats were exposed to the unpredictable chronic stress procedure ${ }^{[19]}$ for 3 weeks. The stressors included food deprivation for $24 \mathrm{~h}$, water deprivation for $24 \mathrm{~h}$, tail pinch ( $2 \mathrm{~cm}$ apart from the end of the tail) for $5 \mathrm{~min}$, heat in an oven at $45^{\circ} \mathrm{C}$ for $5 \mathrm{~min}$, cold swimming at $4-8^{\circ} \mathrm{C}$ for $4 \mathrm{~min}$ and then towel-drying, reverse day and night cycles, and social isolation (one rat per cage) for $24 \mathrm{~h}$. Each stressor was administered randomly once daily per one rat on every $7 \mathrm{~d}$ (three times within $21 \mathrm{~d}$ ) between 8:30 AM and 10:30 $\mathrm{AM}$, except for the 24-h stressors. In other words, there were $7 \mathrm{~d}$ in a cycle, and 7 different stressors were administered randomly in one cycle to ensure that each stressor would be unpredictable for the animals. On the last day of the stress period (PW20), after $12 \mathrm{~h}$ fasting, all rats were obliged to swim at $4-8^{\circ} \mathrm{C}$ for $4 \mathrm{~min}$ and were then towel-dried. One hour after swimming, the rats were anesthetized with isoflurane and decapitated in a room separate from the one where the other animals were kept. Blood samples were collected via the vena caudalis within $5 \mathrm{~min}$ before and after chronic stress, and the serum was prepared by centrifugation at $17205 \times \mathrm{g}$ for $15 \mathrm{~min}$ at $4{ }^{\circ} \mathrm{C}$ and then stored at $-80^{\circ} \mathrm{C}$ until it was used to measure the levels of ACTH, CORT, glucose, insulin, TCH, TG, and FFA. The hypothalamus, pituitary gland and liver were dissected and fixed in a $4 \%$ paraformaldehyde solution for histological examination.

\section{Analysis for blood samples}

Serum ACTH and CORT were detected using radioimmunoassay and an ELISA kit, respectively, following the manufacturer's protocol. The limits of detection for ACTH and CORT are $5 \mathrm{pg} / \mathrm{mL}$ and $0.39 \mathrm{ng} / \mathrm{mL}$, respectively. The levels of serum glucose, TCH, TG and FFA were detected using biochemical assay kits following the manufacturer's protocol.
Serum insulin was determined using a radioimmunoassay kit. The gain rates of serum ACTH and CORT, as well as the insulin resistant index (IRI), were calculated as described below, according to our previous studies ${ }^{[14]}$ :

$\mathrm{ACTH}(\mathrm{CORT})$ con.gain rate $(\%)=$

$\frac{\mathrm{ACTH}(\mathrm{CORT}) \text { con.after stress-ACTH}(\mathrm{CORT}) \text { con.before stress }}{\mathrm{ACTH}(\mathrm{CORT}) \text { con.before stress }} \times 100$

IRI=fasting serum insulin (FINS) $\times$ fasting blood glucose $(\mathrm{FBG}) / 22.5$

\section{Histological examination}

The hypothalamus, pituitary gland and liver were fixed in a $4 \%$ paraformaldehyde solution overnight and processed using the paraffin section technique. Sections that were approximately $5 \mu \mathrm{m}$ thick were stained with hematoxylin and eosin (HE) dyes and observed under a light microscope.

\section{Statistical analysis}

Excel (Microsoft, Redmond, WA, USA) and Prism (GraphPad Software, La Jolla, CA, USA) were used to perform data analysis. All data presented are expressed as the mean \pm SEM. Student's two-tailed $t$-test was used to compare the mean values of various groups as applicable. The paired $t$-test was used to compare the mean values of the same group before and after chronic stress. Statistical significance was designated at $P<0.05$.

\section{Results}

\section{Body weight change after birth}

The rat IUGR model induced by PNE was reported in our previous study ${ }^{[13,14]}$. In the present study, prenatal exposure to nicotine $\left(2 \mathrm{mg} \cdot \mathrm{kg}^{-1} \cdot \mathrm{d}^{-1}\right)$ resulted in a lower body weight compared with that of the control group $(P<0.01$, Figure $2 \mathrm{~A})$ at PW1 (the dams are highly sensitive to environmental stimulus over a fixed time course after delivery, so we weighed the neonates at PW1 but not PD1).

"Catch-up" growth is rapid growth in infants or young children who were born small for their gestational age, and it may be defined as a height velocity that surpasses the statistical limits of normality for age and/or maturity over a defined interval of time, following a transient period of growth inhibition $^{[22]}$. Our subsequent data showed that when compared with the control, the body weight gain rates showed a trend toward increasing or increased significantly, especially at PW16 in the males and from PW11 to PW16 in the female rats $(P<0.05, P<0.01$, Figure $2 B$ and $2 C)$, indicating that these pups exhibited a "catch-up" growth pattern. Moreover, the timing of catch-up growth is late (post-weaning) and genderdependent.

\section{Serum ACTH and CORT concentration changes before and after} chronic stress

Before chronic stress, serum ACTH and CORT concentrations in male and female IUGR offspring were significantly 

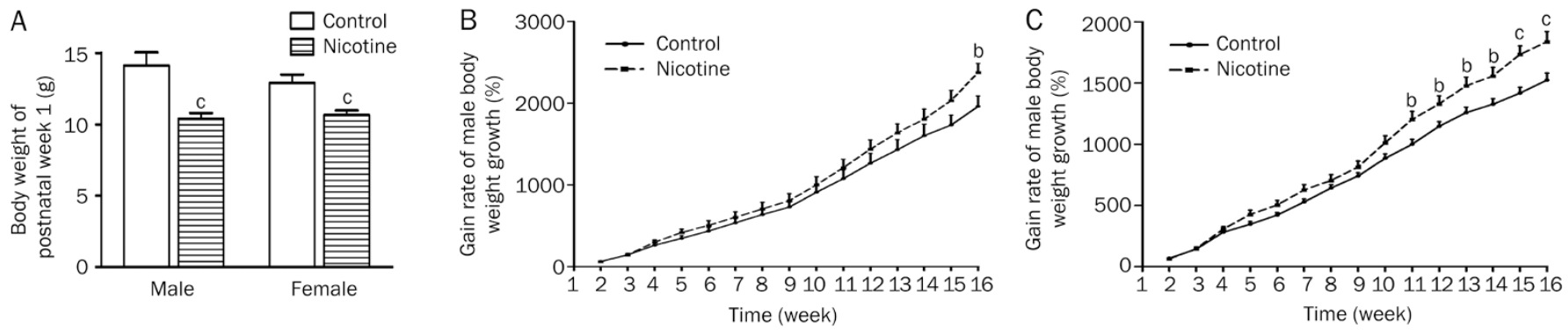

Figure 2. Effects of prenatal nicotine $\left(2 \mathrm{mg} \cdot \mathrm{kg}^{-1} \cdot \mathrm{d}^{-1}\right)$ exposure and a high-fat diet after weaning on the body weight of the offspring rats. (A) Body weight of the offspring at PW1; (B) body weight growth rate in males; (C) body weight growth rate in females. Mean \pm SEM. $n=8$. ${ }^{b} P<0.05,{ }^{\mathrm{C}} P<0.01 \mathrm{vs}$ control.

lower (ACTH: 37\% and 42\% lower than the control for male and female, respectively; CORT: $27 \%$ and $31 \%$ lower than the control for male and female, respectively) $(P<0.01$, Figures $3 \mathrm{~A}, 3 \mathrm{~B}, 3 \mathrm{D}$, and $3 \mathrm{E})$. After chronic stress, the serum ACTH and CORT concentrations were significantly increased in all groups compared to levels before chronic stress $(P<0.01$, Figures $3 \mathrm{~A}, 3 \mathrm{~B}, 3 \mathrm{D}$, and $3 \mathrm{E}$ ). Furthermore, the ACTH levels in both male and female IUGR offspring and the CORT levels in female IUGR offspring were significantly higher than those in the control groups after chronic stress $(P<0.01$, Figures $3 \mathrm{~A}$, $3 \mathrm{~B}$, and $3 \mathrm{E})$. The serum ACTH and CORT concentrations in the PNE groups increased significantly when compared with the control $(P<0.05, P<0.01$, Figures $3 \mathrm{C}$ and $3 \mathrm{~F})$. These results indicated that PNE could enhance the sensitivity of the HPA axis to chronic stress in IUGR offspring fed a high-fat diet.

Serum glucose and insulin concentration alterations before and after chronic stress

Before chronic stress, the fasting serum glucose concentrations
(Figures 4A and 4D) were the same in the PNE and control groups. After chronic stress, the serum glucose concentrations of the PNE rats were significantly increased from baseline $(P<0.01$, Figure $4 \mathrm{~A})$ and were significantly higher than those of the controls $(P<0.05$, Figure $4 \mathrm{~A})$ in males, but no change was observed in the female offspring (Figure 4D). Compared to the control groups, the serum insulin concentrations (Figures $4 \mathrm{~B}$ and $4 \mathrm{E}$ ) before chronic stress were decreased significantly in the male PNE group $(P<0.01$, Figure $4 \mathrm{~B})$ but showed no change in the female PNE group (Figure 4E). After chronic stress, the serum insulin concentrations were significantly increased over baseline $(P<0.01$, Figure $4 \mathrm{~B}$ and $4 \mathrm{E})$ in the female controls and in the male and female PNE rats. Furthermore, the IRI in the male PNE group was significantly lower than that in the control group $(P<0.05$, Figure $4 \mathrm{C})$ before chronic stress, but it increased significantly after chronic stress $(P<0.01)$ and was much higher than that of the control group $(P<0.05)$ (Figure 4 C). The IRI in the female PNE group was similar to that of the control before and after chronic stress,
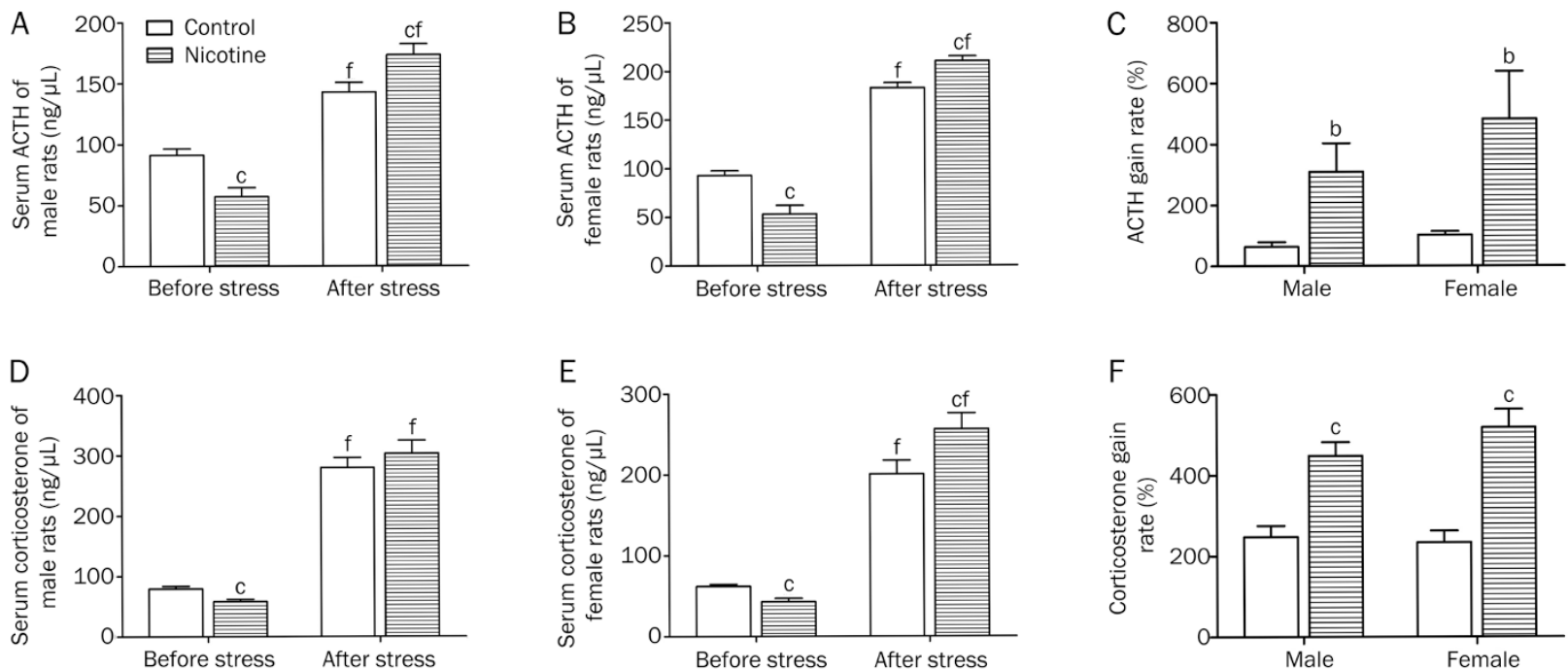

Figure 3. Effects of prenatal nicotine $\left(2 \mathrm{mg} \cdot \mathrm{kg}^{-1} \cdot \mathrm{d}^{-1}\right)$ exposure and a high-fat diet after weaning on the concentrations of serum adrenocorticotrophic hormone $(\mathrm{ACTH})$ and corticosterone in adult offspring rats before and after chronic stress. (A) Serum ACTH concentrations of male rats before and after stress; (B) serum ACTH concentrations of female rats before and after stress; (C) ACTH rates of increase in male and female rats; (D) serum corticosterone concentrations of male rats before and after stress; (E) serum corticosterone concentrations of female rats before and after stress; ( $F$ ) corticosterone rates of increase in male and female rats. Mean \pm SEM. $n=8 .{ }^{b} P<0.05,{ }^{\circ} P<0.01$ vs control. ${ }^{f} P<0.01$ vs before stress. 

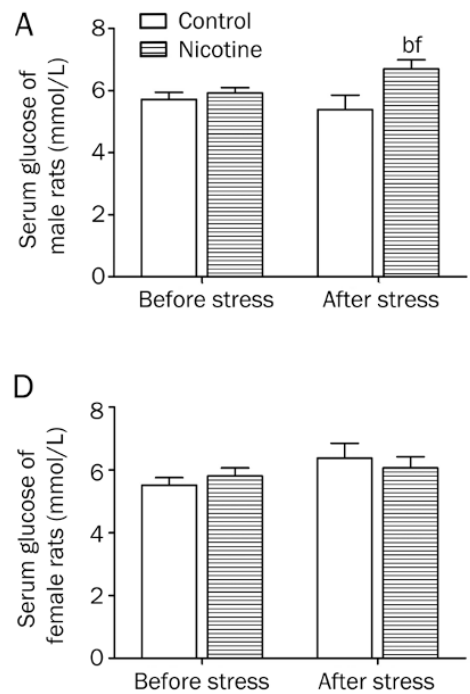
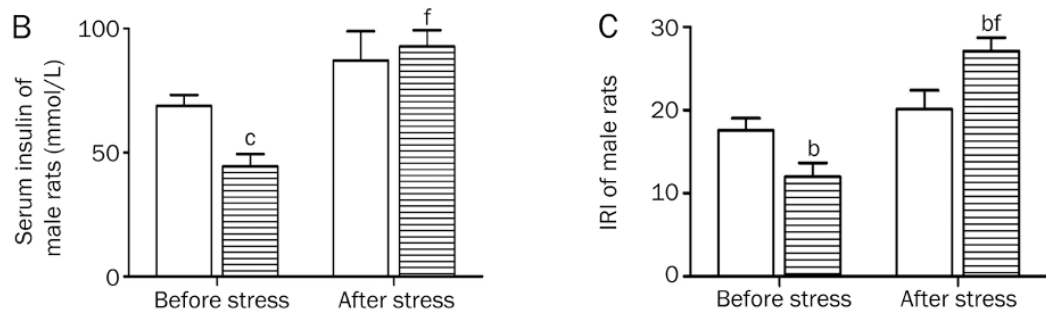

E

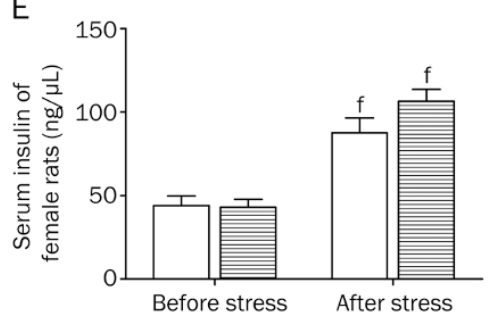

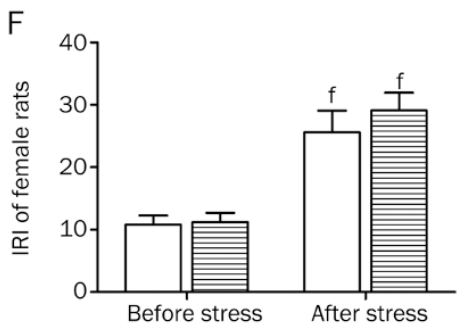

Figure 4. Effects of prenatal nicotine $\left(2 \mathrm{mg} \cdot \mathrm{kg}^{-1} \cdot \mathrm{d}^{-1}\right)$ exposure and a high-fat diet after weaning on the levels of serum glucose and insulin in adult offspring rats before and after chronic stress. (A) Serum glucose of males; (B) serum insulin of males; (C) IRI of males; (D) serum glucose of females; (E) serum insulin of females; (F) IRI of females. Mean \pm SEM. $n=8$. ${ }^{b} P<0.05,{ }^{\mathrm{C}} P<0.01$ vs control. ${ }^{\mathrm{f}} P<0.01$ vs before stress.

but showed a marked increase over the baseline after chronic stress $(P<0.01$, Figure $4 \mathrm{~F})$.

\section{Blood lipid alterations before and after chronic stress}

The serum TCH, TG, and FFA levels in the male PNE group were similar or much higher $(P<0.01)$ than that of the control (Figures 5A, 5C, and 5E) before chronic stress. After chronic stress, the TG and TCH levels in the male PNE rats showed a tendency toward increasing compared to those of control (Figures 5A and 5C), whereas the serum FFA level significantly decreased compared with the level before chronic stress $(P<0.01$, Figure $5 \mathrm{E})$. In the PNE female rats, the serum TCH and FFA levels were similar to those of the control rats (Fig- ures $5 \mathrm{~B}$ and $5 \mathrm{~F}$ ), whereas the serum TG level was much lower than that of the control before chronic stress. After chronic stress, the TCH and TG levels in the PNE female rats remained unchanged compared with those of the control (Figure 5B and $5 \mathrm{D})$, whereas the FFA level significantly decreased compared with the level before chronic stress $(P<0.01$, Figure 5F).

\section{Pathological alterations}

The normal architecture of the hypothalamus, pituitary gland and liver were observed in the male and female control rats (Figures 6A1-6A3 and 6C1-6C3). In contrast, many lipid droplets were observed in the hypothalamus, pituitary gland and liver tissues of the PNE rats (Figures 6B1-6B3, 6D1-6D3),
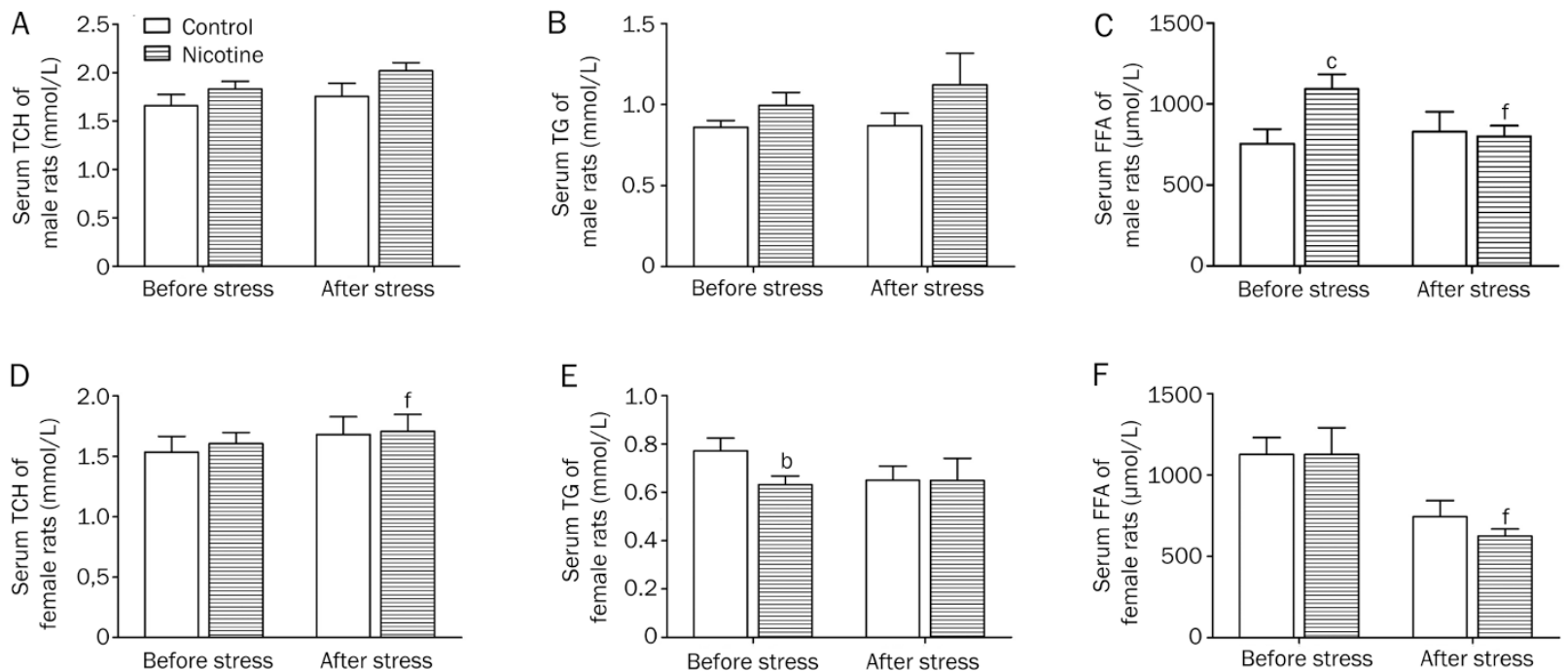

Figure 5. Effects of prenatal nicotine $\left(2 \mathrm{mg} \cdot \mathrm{kg}^{-1} \cdot \mathrm{d}^{-1}\right)$ exposure and a high-fat diet after weaning on the levels of blood lipids in adult offspring rats before and after chronic stress. (A) Serum total cholesterol (TCH) of males; (B) serum triglyceride (TG) of males; (C) serum free fatty acids (FFA) of males; (D) serum TCH of females; (E) serum TG of females; (F) serum FFA of females. Mean \pm SEM. $n=8 .{ }^{b} P<0.05,{ }^{c} P<0.01$ vs control. ${ }^{f} P<0.01$ vs before stress. 
particularly in the female rats. These results reveal a wide range of abnormal intracellular retention of lipids in the majority of neuroendocrine metabolic-related tissues of IUGR rats.

\section{Discussion}

PNE induces low birthweight and typical "catch-up" growth in rats fed a high-fat diet

The phenomenon of low birthweight as a result of PNE in rats has been previously described ${ }^{[23-25]}$. A series of epidemiological, clinical and experimental studies performed throughout the world have demonstrated that there is an association among slow growth before birth; accelerated growth in early postnatal life; and the emergence of glucose intolerance, insulin resistance, type 2 diabetes and obesity in later life ${ }^{[26-29]}$. Our previous studies have found that the IUGR offspring with PNE who were fed a normal diet failed to present a significant "catch up" growth pattern of body weight ${ }^{[14]}$. However, the present study demonstrated that IUGR offspring, particularly females, that were induced by PNE exhibited a lower birthweight and presented a "catch-up" growth pattern and that the catch-up timing is late (post-weaning) and gender-dependent. The results were consistent with Fowden's hypothesis, which suggested that this "catch-up growth" pattern after birth was due to an increased sensitivity of the peripheral tissues to metabolic hormones induced by suboptimal conditions in utero $^{[7]}$.
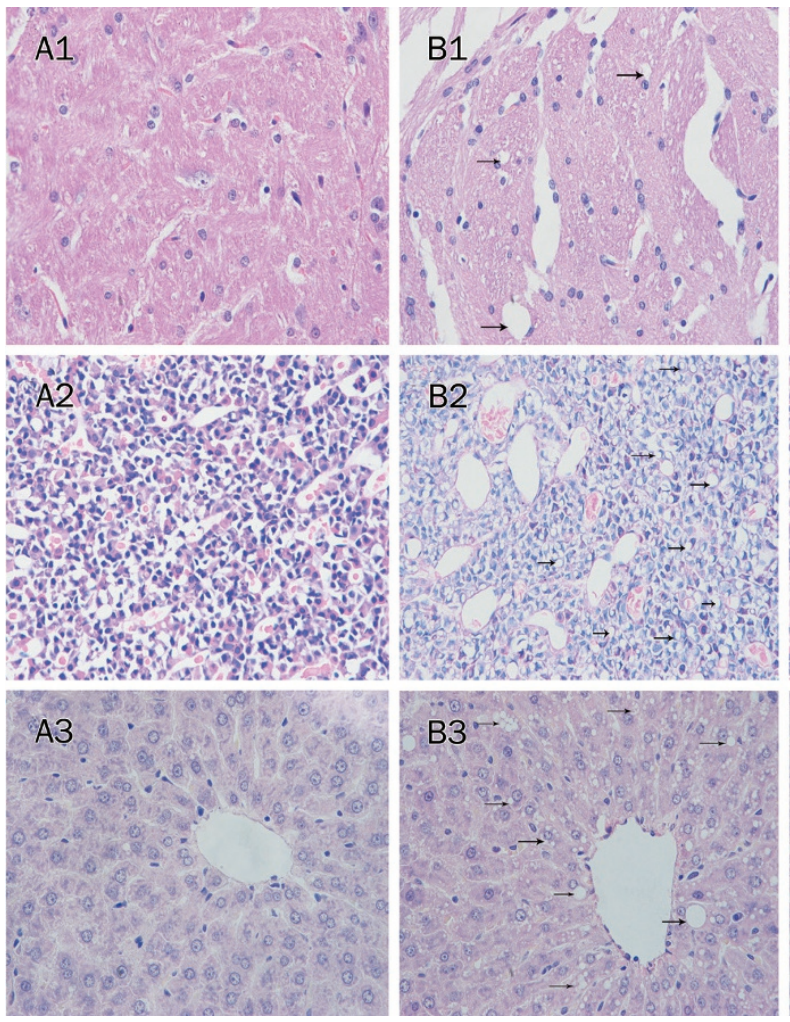

The studies to date have reported increased fat accumulation after catch-up growth in low birthweight infants and in animal models of IUGR ${ }^{[30,31]}$. Previous studies in rats have demonstrated that the majority of body fat is located in subcutaneous deposits at the time of weaning (subcutaneous fat weight increases 6-fold). Two months later, the majority of body fat is located in the abdominal cavity (intra-abdominal fat increases 116-fold $)^{[32]}$. Therefore, it can be assumed that the "catch-up" growth of IUGR pups after weaning may contribute to the greater accumulation of abdominal fat and favor a later-stage metabolic dysfunction in IUGR rats. In the present study, "catch-up growth" in the body weight of female IUGR offspring occurred after PW11, so the incremental increases in body weight were likely mostly abdominal fat, which might accelerate the development of MS.

PNE aggravates the programed alteration of the HPA axis for lower basal activity and enhanced stress sensitivity in the context of a high-fat diet

It has been suggested that the intrauterine programming of the HPA axis is most likely involved in the underlying mechanism behind the intrauterine origin of adult $\mathrm{MS}^{[33,34]}$. Our previous studies suggested that PNE induced a program of changes in the HPA axis, which was manifested as lower basal activity and enhanced stress sensitivity of the HPA axis ${ }^{[13,14]}$. Other studies showed that a high-fat diet increased the basal activity

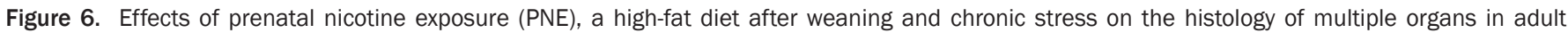

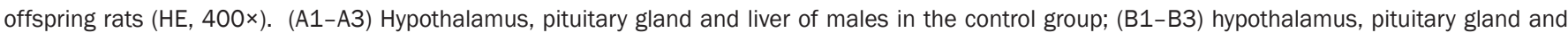

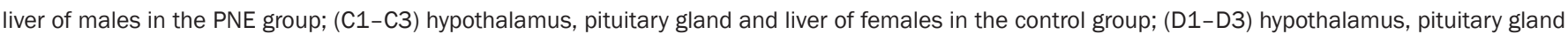
and liver of females in the PNE group. 
of the HPA axis in rats by promoting the synthesis and secretion of CRH in the hypothalamic paraventricular nucleus ${ }^{[35,36]}$. However, in the present study, before chronic stress, blood ACTH and CORT levels in the IUGR offspring rats fed a highfat diet remained much lower. In speculating, this lower basal activity of the HPA axis might be primarily attributed to the lowered programming of the intrauterine HPA axis induced by PNE. Moreover, PNE seemed to aggravate this programmed effect in the context of a high-fat diet. However, the underlying mechanisms require further study.

The major neuroendocrine response mediating stress adaptation is the activation of the HPA axis leading to increased circulatory ACTH and GC levels ${ }^{[37,38]}$. As reported in the literature $^{[39]}$, the serum CORT concentration increased dramatically in offspring with $72 \mathrm{~h}$ of prenatal nutrition restriction and liquid deprivation. In the present study, after the unpredictable chronic stress, the levels of blood ACTH, CORT and their rates of increase were remarkably higher in the PNE group than those of the control group. These results suggested that PNE enhanced the sensitivity of the HPA axis to chronic stress. Moreover, a high-fat diet can also increase the release of ACTH and CORT after stress in adult rats ${ }^{[0]}$. Therefore, in the present study, the enhanced sensitivity of the HPA axis to chronic stress in adult offspring might result from the potentiation of PNE and the postnatal high-fat diet.

PNE aggravated the effect of the programmed HPA axis on changes in the glucose and lipid metabolism in the context of a high-fat diet

Reynolds suggested that a high level of circulatory GC in utero may be vital for the intrauterine programming of glucose and lipid metabolism ${ }^{[41]}$. We previously confirmed that PNE induced a HPA axis-associated neuroendocrine metabolic alteration in IUGR fetal rats, which manifested as a high blood glucose level and low blood lipid levels (TG and TCH) associated with a high blood GC level ${ }^{[13]}$. After birth and the initiation of a normal diet, the level of blood glucose in IUGR offspring showed no change before chronic stress but increased significantly after chronic stress, and the level of blood TCH and TG were much higher than those in the control group before chronic stress but decreased as compared to the control after chronic stress ${ }^{[14]}$.

Early metabolic programming could contribute to the observed variability in responses to nutritional interventions and further the development of metabolic diseases in later life, particularly in environments where high-fat diets are prevalent. A high-fat diet has been used to induce obesity, dyslipidemia and IR in rodents for many decades. The complications developed by high-fat diet resemble human MS, and these complications may extend to cardiac hypertrophy, cardiac fibrosis, myocardial necrosis and hepatic steatosis ${ }^{[42-45]}$. In the present study, we found that after chronic stress, serum glucose and insulin concentrations and the IRI were significantly increased in male IUGR offspring, but the phenomenon failed to appear in IUGR females, indicating that the male offspring of PNE group presented hyperglycemia and IR.
We also found that before chronic stress, the serum $\mathrm{TCH}$ and TG concentrations in the PNE group were slightly or significantly increased (except for TG in females), but they still showed a tendency toward increasing after chronic stress. These results were not in agreement with those of our previous study in which the offspring were fed a normal diet ${ }^{[14]}$, indicating that the differences could possibly be attributed to the high-fat diet. It is known that FFA is an intermediate product of lipid synthesis and catabolism. However, we found that serum FFA concentrations in the PNE group were decreased significantly after chronic stress, suggesting that lipid catabolism remained enhanced and may be evoked by excessive circulatory GC.

Moreover, certain distinctive pathological phenomenon, such as extensive accumulation of large lipid droplets, vacuolar degeneration (steatosis) and even cellular necrosis were observed in the HPA axis-associated neuroendocrine metabolic organs (hypothalamus, pituitary gland and liver) in the PNE group, indicating the occurrence of lipoidosis. Combined with hyperglycemia, IR and lipoidosis, the programmed neuroendocrine metabolic effects of PNE and a high-fat diet may be the most important cause for these metabolic-related diseases.

\section{PNE induced gender-specific neuroendocrine metabolic changes in the context of a high-fat diet}

Interestingly, in the present study, gender differences were observed in the adult IUGR offspring fed a high-fat diet. For example, the "catch-up" pattern of body weight growth was obvious, especially in the female offspring. This result was consistent with that of a previous epidemiological report that revealed the high prevalence of overweight in adult women exposed to the Dutch or Chinese famine during their fetal life ${ }^{[46,47]}$. Furthermore, the serum insulin concentration was remarkably lower before chronic stress but increased significantly in the males after stress; however, although the serum glucose concentration was much higher than that of the control after stress, this phenomenon was not observed in females. Moreover, compared with the control, there were higher levels of lipids (especially TG and FFA) in males but not in females. According to Pinheiro's report ${ }^{[48]}$, we speculate that the obvious "catch-up" growth pattern of body weight in the female offspring may reflect a leptin production impairment in the females, and the changes in insulin/glucose/lipids in the males may reflect that the male offspring rats were more sensitive to the stress/GC that resulted from the effects on adrenal function. Another possible source of the gender differences might be sexual hormones. Recent studies suggest that sex hormones may contribute to sexual dimorphisms that occur in response to fetal insult ${ }^{[49,50]}$. Our recent data suggest that the estrogen level was lower in female rats exposed to ethanol prenatally than in control female rats, but no significantly differences were observed between the two male groups (our unpublished data). In addition, examining the effects of a high-fat diet on metabolism revealed gender-dependent changes in the levels of key components of the insulin and adi- 
ponectin signaling pathways ${ }^{[51]}$, suggesting a different effect of a high-fat diet on male versus female rats.

\section{Conclusions}

Our previous studies have confirmed that PNE induced the developmental dysfunction of the HPA axis and associated neuroendocrine metabolic programmed changes in IUGR adult offspring rats. In this study, we further demonstrated that PNE could induce typical "catch-up" growth, aggravate the programmed HPA-associated neuroendocrine metabolic changes, and enhance the susceptibility to metabolic diseases in adult IUGR offspring rats fed a high-fat diet. Moreover, some of these effects presented in a gender-specific manner. These observed phenomena and their suggesting mechanisms may further provide an experimental basis for explaining the susceptibility to adult metabolic diseases in IUGR offspring. Moreover, the results further our understanding of the harm of nutritional intervention on IUGR offspring for preventing and/or retarding the development of adult metabolic diseases.

\section{Acknowledgements}

This work was supported by grants from the National Natural Science Foundation of China (№ 30830112, 81072709, 81220108026, and 81371483) and the Key Grant Project of the Chinese Ministry of Education (№ V200801).

\section{Author contribution}

Dan XU, Li-ping XIA, Jacques MAGDALOU, and Hui WANG conceived and designed the experiments; Dan XU, Li-ping XIA, Ben-jian ZHANG, Lang SHEN, and You-ying LEI performed the experiments; Dan XU, Li-ping XIA, Lian LIU, Li ZHANG, and Hui WANG analyzed the data; Jacques MAGDALOU and Hui WANG contributed reagents/materials/ analysis tools; Dan XU, Li-ping XIA, and Hui WANG wrote the paper.

\section{References}

1 Ford ES, Li C, Zhao G. Prevalence and correlates of metabolic syndrome based on a harmonious definition among adults in the US. J Diabetes 2010; 2: 180-93.

2 Gu DF, Reynolds K, Yang WJ, Chen SF, Wu XG, Duan XF, et al. The prevalence of metabolic syndrome in the general adult population aged 35-74 years in China. Chin J Diabetes 2005; 13: 181-6.

3 Silveira VM, Horta BL. Birth weight and metabolic syndrome in adults: meta-analysis. Rev Saude Publica 2008; 42: 10-8.

4 Zhuang YL. Morden obstetrics. Beijing: Science press; 2003. p 384.

5 Alisi A, Panera N, Agostoni C, Nobili V. Intrauterine growth retardation and nonalcoholic Fatty liver disease in children. Int J Endocrinol 2011; 2011: 269853.

6 Fowden AL, Forhead AJ, Coan PM, Burton GJ. The placenta and intrauterine programming. J Neuroendocrinol 2008; 20: 439-50.

7 Fowden AL, Giussani DA, Forhead AJ. Endocrine and metabolic programming during intrauterine development. Early Hum Dev 2005; 81: 723-34.

8 Yildiz D. Nicotine, its metabolism and an overview of its biological effects. Toxicon 2004; 43: 619-32.

9 Quigley ME, Sheehan KL, Wilkes MM, Yen SS. Effects of maternal smoking on circulating catecholamine levels and fetal heart rates. Am J Obstet Gynecol 1979; 133: 685-90.

10 Slotkin TA. Fetal nicotine or cocaine exposure: which one is worse? J Pharmacol Exp Ther 1998; 285: 931-45.

11 Czekaj P, Palasz A, Lebda-Wyborny T, Nowaczyk-Dura G, Karczewska $\mathrm{W}$, Florek $\mathrm{E}$, et al. Morphological changes in lungs, placenta, liver and kidneys of pregnant rats exposed to cigarette smoke. Int Arch Occup Environ Health 2002; 75 Suppl: S27-35.

12 Nelson E, Goubet-Wiemers C, Guo Y, Jodscheit K. Maternal passive smoking during pregnancy and foetal developmental toxicity. Part 2: histological changes. Hum Exp Toxicol 1999; 18: 257-64.

13 Xu D, Liang G, Yan YE, He WW, Liu YS, Chen LB, et al. Nicotineinduced over-exposure to maternal glucocorticoid and activated glucocorticoid metabolism causes hypothalamic-pituitary-adrenal axis-associated neuroendocrine metabolic alterations in fetal rats. Toxicol Lett 2012; 209: 282-90.

14 Liu L, Liu F, Kou H, Zhang BJ, Xu D, Chen B, et al. Prenatal nicotine exposure induced a hypothalamic-pituitary-adrenal axis-associated neuroendocrine metabolic programmed alteration in intrauterine growth retardation offspring rats. Toxicol Lett 2012; 214: 307-13.

15 Langdon KD, Clarke J, Corbett D. Long-term exposure to high fat diet is bad for your brain: exacerbation of focal ischemic brain injury. Neuroscience 2011; 182: 82-7.

16 Touati S, Meziri F, Devaux S, Berthelot A, Touyz RM, Laurant P. Exercise reverses metabolic syndrome in high-fat diet-induced obese rats. Med Sci Sports Exerc 2011; 43: 398-407.

17 Panchal SK, Brown L. Rodent models for metabolic syndrome research. J Biomed Biotechnol 2011; 2011: 351982.

18 Fraulob JC, Ogg-Diamantino R, Fernandes-Santos C, Aguila MB, Mandarim-de-Lacerda CA. A mouse model of metabolic syndrome: insulin resistance, fatty liver and non-alcoholic fatty pancreas disease (NAFPD) in C57BL/6 mice fed a high fat diet. J Clin Biochem Nutr 2010; 46: 212-23.

19 Willner P. Chronic mild stress (CMS) revisited: consistency and behavioural-neurobiological concordance in the effects of CMS. Neuropsychobiology 2005; 52: 90-110.

20 Manuel-Apolinar L, Zarate A, Rocha L, Hernandez M. Fetal malnutrition affects hypothalamic leptin receptor expression after birth in male mice. Arch Med Res 2010; 41: 240-5.

21 Wang $\mathrm{H}$, Liao ZX, Chen M, Hu XL. Effects of hepatic fibrosis on ofloxacin pharmacokinetics in rats. Pharmacol Res 2006; 53: 28-34.

22 Boersma B, Wit JM. Catch-up growth. Endocr Rev 1997; 18: 646-61.

23 Suzuki K, Tanaka T, Kondo N, Minai J, Sato M, Yamagata Z. Is maternal smoking during early pregnancy a risk factor for all low birth weight infants? J Epidemiol 2008; 18: 89-96.

24 Kinzler WL, Kaminsky L. Fetal growth restriction and subsequent pregnancy risks. Semin Perinatol 2007; 31: 126-34.

25 Romo A, Carceller R, Tobajas J. Intrauterine growth retardation (IUGR): epidemiology and etiology. Pediatr Endocrinol Rev 2009; 6 Suppl 3: 332-6.

26 Langley-Evans SC. Nutritional programming of disease: unravelling the mechanism. J Anat 2009; 215: 36-51.

27 Yajnik CS. Nutrient-mediated teratogenesis and fuel-mediated teratogenesis: two pathways of intrauterine programming of diabetes. Int J Gynaecol Obstet 2009; 104 Suppl 1: S27-31.

28 Briana DD, Malamitsi-Puchner A. Intrauterine growth restriction and adult disease: the role of adipocytokines. Eur J Endocrinol 2009; 160: $337-47$.

29 Hales CN, Barker DJ. Type 2 (non-insulin-dependent) diabetes mellitus: the thrifty phenotype hypothesis. Diabetologia 1992; 35 : 595-601. 
30 Bol VV, Delattre Al, Reusens B, Raes M, Remacle C. Forced catch-up growth after fetal protein restriction alters the adipose tissue gene expression program leading to obesity in adult mice. Am J Physiol Regul Integr Comp Physiol 2009; 297: R291-9.

31 Isganaitis E, Jimenez-Chillaron J, Woo M, Chow A, DeCoste J, Vokes $\mathrm{M}$, et al. Accelerated postnatal growth increases lipogenic gene expression and adipocyte size in low-birth weight mice. Diabetes 2009; 58: 1192-200.

32 Caluwaerts S, Lambin S, van Bree R, Peeters H, Vergote I, Verhaeghe J. Diet-induced obesity in gravid rats engenders early hyperadiposity in the offspring. Metabolism 2007; 56: 1431-8.

33 Kanaka-Gantenbein C. Fetal origins of adult diabetes. Ann N Y Acad Sci 2010; 1205: 99-105.

34 Xita N, Tsatsoulis A. Fetal origins of the metabolic syndrome. Ann N Y Acad Sci 2010; 1205: 148-55.

35 Chan O, Inouye K, Riddell MC, Vranic M, Matthews SG. Diabetes and the hypothalamo-pituitary-adrenal (HPA) axis. Minerva Endocrinol 2003; 28: 87-102.

36 Shin AC, MohanKumar SM, Sirivelu MP, Claycombe KJ, Haywood JR, Fink GD, et al. Chronic exposure to a high-fat diet affects stress axis function differentially in diet-induced obese and diet-resistant rats. Int J Obes (Lond) 2010; 34: 1218-26.

37 Tsigos C, Chrousos GP. Hypothalamic-pituitary-adrenal axis, neuroendocrine factors and stress. J Psychosom Res 2002; 53: 865-71.

$38 \mathrm{Fu} \mathrm{JH}$, Xie SR, Kong SJ, Wang Y, Wei W, Shan Y, et al. The combination of a high-fat diet and chronic stress aggravates insulin resistance in Wistar male rats. Exp Clin Endocrinol Diabetes 2009; 117: 354-60.

39 Vieau D, Sebaai N, Leonhardt M, Dutriez-Casteloot I, Molendi-Coste O, Laborie $\mathrm{C}$, et al. HPA axis programming by maternal undernutrition in the male rat offspring. Psychoneuroendocrinology 2007; 32 Suppl 1: S16-20.

40 Tannenbaum BM, Brindley DN, Tannenbaum GS, Dallman MF, McArthur MD, Meaney MJ. High-fat feeding alters both basal and stress-induced hypothalamic-pituitary-adrenal activity in the rat. Am J Physiol 1997; 273: E1168-77.

41 Reynolds RM. Corticosteroid-mediated programming and the pathogenesis of obesity and diabetes. J Steroid Biochem Mol Biol
2010; 122: 3-9.

42 Woods SC, Seeley RJ, Rushing PA, D'Alessio D, Tso P. A controlled high-fat diet induces an obese syndrome in rats. J Nutr 2003; 133 : 1081-7.

43 Aguila MB, Mandarim-de-Lacerda CA. Heart and blood pressure adaptations in Wistar rats fed with different high-fat diets for 18 months. Nutrition 2003; 19: 347-52.

44 Kobayasi R, Akamine EH, Davel AP, Rodrigues MA, Carvalho CR, Rossoni LV. Oxidative stress and inflammatory mediators contribute to endothelial dysfunction in high-fat diet-induced obesity in mice. J Hypertens 2010; 28: 2111-9.

45 Buettner R, Parhofer KG, Woenckhaus M, Wrede CE, Kunz-Schughart LA, Scholmerich J, et al. Defining high-fat-diet rat models: metabolic and molecular effects of different fat types. J Mol Endocrinol 2006; 36: 485-501.

46 Ravelli AC, van Der Meulen JH, Osmond C, Barker DJ, Bleker OP. Obesity at the age of $50 \mathrm{y}$ in men and women exposed to famine prenatally. Am J Clin Nutr 1999; 70: 811-6.

47 Wang Y, Wang X, Kong Y, Zhang JH, Zeng Q. The Great Chinese Famine leads to shorter and overweight females in Chongqing Chinese population after 50 years. Obesity (Silver Spring) 2010; 18 : 588-92.

48 Pinheiro CR, Oliveira E, Trevenzoli IH, Manhaes AC, Santos-Silva AP, Younes-Rapozo V, et al. Developmental plasticity in adrenal function and leptin production primed by nicotine exposure during lactation: gender differences in rats. Horm Metab Res 2011; 43: 693-701.

49 Ojeda NB, Grigore D, Yanes LL, Iliescu R, Robertson EB, Zhang H, et al. Testosterone contributes to marked elevations in mean arterial pressure in adult male intrauterine growth restricted offspring. Am J Physiol Regul Integr Comp Physiol 2007; 292: R758-63.

50 Ojeda NB, Grigore D, Robertson EB, Alexander BT. Estrogen protects against increased blood pressure in postpubertal female growth restricted offspring. Hypertension 2007; 50: 679-85.

51 Amengual-Cladera E, Llado I, Gianotti M, Proenza AM. Sex differences in the effect of high-fat diet feeding on rat white adipose tissue mitochondrial function and insulin sensitivity. Metabolism 2012; 61: 1108-17. 